The Astrophysical Journal SuPPlement Series, 90:521-530, 1994 February

(1) 1994. The American Astronomical Society. All rights reserved. Printed in U.S.A.

\title{
SOURCES AND ACCELERATION OF ENERGETIC PARTICLES IN PLANETARY MAGNETOSPHERES
}

\author{
EBERHARD MÖBIUS ${ }^{1}$ \\ Space Science Center, Institute for the Study of Earth, Oceans, and Space, University of New Hampshire, Durham, NH 03824 \\ Received 1993 March 10; accepted 1993 July 9
}

\begin{abstract}
Energetic particles in the magnetospheres of the solar system originate from various different sources, such as the solar wind, the planetary ionospheres as well as the moons and rings of the planetary systems. Important acceleration sites are the auroral regions, the magnetotail, and the equatorial regions of the magnetospheres where electric fields, wave-particle interactions and magnetic pumping are among the major acceleration mechanisms proposed. Over the last decade mass- and charge-sensitive particle spectrometers on satellites and space probes have collected a wealth of information about the relative contribution of the various particle sources and the major acceleration processes to the energetic particle populations. Emphasis will be put on recent studies of the source populations and the acceleration processes in the Earth's auroral zones and magnetotail. Furthermore, the Jovian system with the largest magnetosphere and its unique mixture of particle sources with strong contributions from moons will be highlighted in some results from Ulysses.
\end{abstract}

Subject headings: acceleration of particles — planets and satellites: general

\section{INTRODUCTION}

Planetary magnetospheres play a central role in the context of astrophysical particle acceleration mechanisms. First, it is well-known since the early days of space flight that the Earth's magnetosphere is a powerful source of energetic particles. This view has been widened to include all magnetospheres in the solar system by a variety of successful planetary missions. Secondly, the Earth's magnetosphere is the most intensely studied astrophysical laboratory, because of its proximity and the relatively easy access by means of Earth-orbiting satellites. Finally, planetary magnetospheres constitute models for more remote and exotic objects, such as the magnetospheres of pulsars, which can only be studied through their emission of electromagnetic radiation. Therefore, a closer look at the planetary magnetospheres in the solar system with special emphasis on the Earth seems to be a very helpful introduction for the discussion of the variety of conditions under which the acceleration of particles is observed in space.

In order to streamline the presentation in this review the discussion will be restricted to magnetospheres of planetary bodies with an intrinsic magnetic field. Therefore, this paper will be limited to the magnetospheres of the Earth, Mercury, Jupiter, Saturn, Uranus, and Neptune. Most emphasis will be put on the Earth and Jupiter, since significantly more information is available on these magnetospheres. The very recent flyby of the Ulysses spacecraft through the Jovian system has provided new exciting data (Smith, Wenzel, \& Page 1992). We will ignore systems, such as Venus and comets, in which the solar wind interacts directly with the atmospheric environment. There will also be no discussion of acceleration at planetary or cometary bow shocks. This field will be covered in separate papers by Sagdeev, Jones, and Krimigis in this issue. Furthermore, we will concentrate mainly on ions and will only turn to electrons where they tell an additional unique story.

\footnotetext{
'E-mail: UNHRCC::MOEBIUS
}

In this contribution the acceleration and the sources of energetic particles will be discussed together. The reason for this choice is threefold:

1. To deduce the amount of acceleration produced by a specific physical process it is necessary to establish the initial source distribution of the particles as a baseline.

2. It has been widely observed that the injection into the acceleration process and the subsequent acceleration itself can be highly selective of particle species and energy.

3. The variety of different particle species offers samples of particles with a wide range in energy/charge $(E / Q)$, energy/ mass $(E / M)$, and magnetic rigidity $\sqrt{(2 m E)} / Q$, parameters which play an important role in different acceleration mechanisms. Thus ion composition provides an excellent tool for the study of their relative importance.

In $\S 2$ we will define the major magnetospheric regions and give a rough scaling of the different magnetospheres. A tour through the main regions of the Earth's magnetosphere will follow in $\S 3$ with a description of the principal magnetospheric acceleration mechanisms and the observed energetic particle distributions. Some important observations in other magnetospheres will be added in $\S 4$. The energetic particle observations in the Jovian system including some of the recent results from the Ulysses mission will be presented in $\S 5$. Section 6 will be devoted to a comprehensive discussion of the commonalities and differences of the sources, acceleration mechanisms, and related dynamics in the various magnetospheres. Finally, we will conclude with a brief summary of the present knowledge and an outlook towards space missions in the foreseeable future. Due to the broad scope the paper must be selective in its coverage of detailed observations and physical mechanisms.

\section{THE STRUCTURE AND SCALING OF MAGNETOSPHERES}

The dynamics of magnetospheres is controlled by the interaction of the solar wind with the magnetic field of a planetary body. Because the solar wind constitutes a highly conductive 
plasma it is mainly excluded from the realm of the planetary magnetic field. The volume of exclusion of the solar wind is the magnetosphere. By this relatively restricted definition we disregard planets with no magnetic field or with a field so weak that it does not reach beyond the atmosphere of the planet, as in the cases of Venus and Mars. A schematic view of the various regions of the Earth's magnetosphere is given in Figure 1. The solar wind flow is diverted around the obstacle of the magnetosphere and makes contact at the magnetopause, the magnetospheric boundary. Because the solar wind flows at supersonic speed, a shock wave, the bow shock, is formed at a certain distance in front of the magnetopause. The particle acceleration observed at the bow shock due to the interaction with the solar wind is discussed in other contributions in this issue. On the downwind side of the Earth the magnetosphere forms the elongated magnetotail which contains regions with substantial particle acceleration due to induced electric fields and with storage of hot plasma in the plasma sheet. The most energetic particle populations in magnetospheres are found trapped in the dipole magnetic field configuration of the ring current and the radiation belts. The regions with significant injection of ionospheric plasma into the magnetospheric system and substantial precipitation of energetic particles into the atmosphere are the polar regions of the magnetosphere, in particular, the auroral zones.

These regions are typical for most of the planetary magnetospheres in the solar system. However, the sizes of these regions and their relative importance for particle acceleration and storage vary substantially with

1. The distance from the Sun, i.e., with the solar wind dynamic pressure, and

2. The dipole moment of the planetary magnetic field.

The size of a magnetosphere can be estimated in the most simple way at the subsolar point of the magnetopause by invoking a balance between the dynamic pressure of the solar wind and the local magnetic pressure:

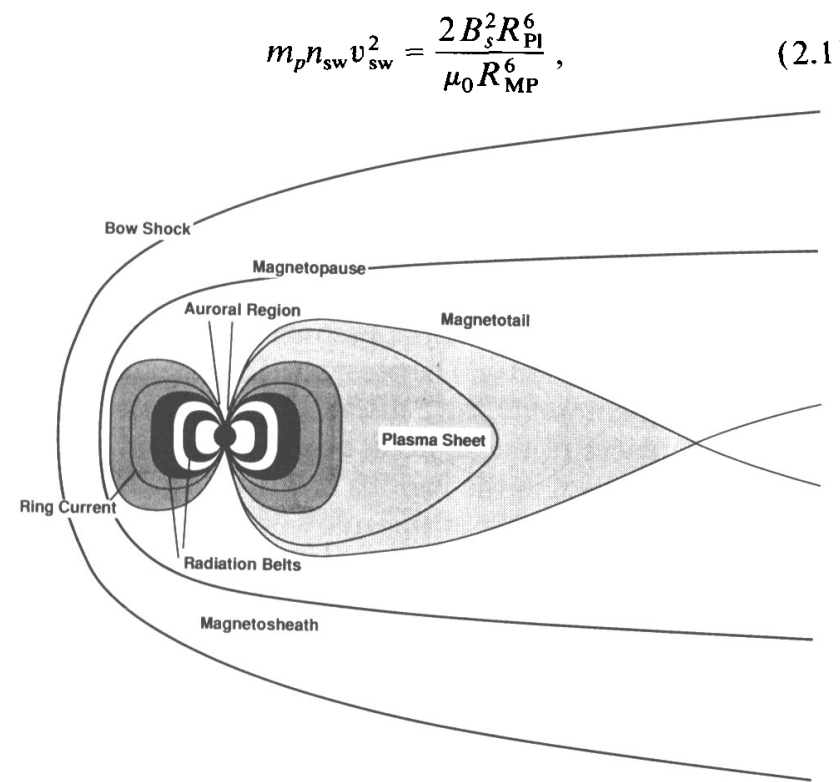

FIG. 1.-Schematics of the Earth's magnetosphere with the key regions for particle acceleration and transport as mentioned in the text. where $n_{\mathrm{sw}}$ and $v_{\mathrm{sw}}$ are the solar wind density and velocity and $B_{s}, R_{\mathrm{PI}}$, and $R_{\mathrm{MP}}$ are the surface magnetic field strength, planetary radius and distance of the magnetopause from the center of the planet. An increase of the local magnetic field by a factor of 2 over the original dipole value at the magnetopause due to the induced current in the boundary has been taken into account in the pressure balance. This leads to a scaling of the magnetopause distance, which may be adopted as a general determination of the size of the magnetosphere:

$$
\frac{R_{\mathrm{MP}}}{R_{\mathrm{Pl}}}=\sqrt[6]{\frac{2 B_{s}^{2}}{\mu_{0} m_{p} n_{\mathrm{sw}} v_{\mathrm{sw}}^{2}}} .
$$

The sizes of the various magnetospheres in the solar system and the relevant parameters are compiled in Table 1. The largest entity in our solar system is the Jovian magnetosphere, whereas Mercury's magnetosphere barely extends to $50 \%$ beyond the surface of the planet. The Earth's magnetosphere is clearly a representative magnetosphere.

\section{A TOUR OF THE EARTH'S MAGNETOSPHERE}

Let us start with the study of the Earth's magnetosphere, at our front doorstep. Numerous spacecraft missions have gathered material about energetic particle populations and their composition in the magnetosphere, with the most detailed observations from spacecraft such as ISEE, DE, AMPTE, and VIKING. From these observations it has been well established that

1. The energetic ions are of solar wind and ionospheric origin.

2. The energy spectra of the ions generally can be best represented in terms of energy per charge.

3. The closer to the earth the more energetic are the particle populations with the exception of some transient events.

Figure 2 shows a sample of energy spectra of ion distributions in the ring current during a magnetospheric storm as taken by the AMPTE CCE CHEM experiment (Gloeckler et al. 1985). Shown is the differential energy density as a function of energy per charge $(\epsilon=E / Q)$. The ring current contains particles which are clearly of solar wind origin $\left(\mathrm{He}^{2+} ; \mathrm{C}, \mathrm{N}\right.$, $\mathrm{O}^{6+}$ ) as well as those of ionospheric origin $\left(\mathrm{O}^{+}\right)$. In addition, charge exchange with the exosphere plays a role in contributing ions such as $\mathrm{He}^{+}$and $\mathrm{O}^{2+}$. Charge exchange processes have also been found to be important for the radial transport, dynamics and particle loss in the ring current (Spjeldvik \& Fritz 1978; Hamilton et al. 1988), and they may produce either

TABLE 1

SIZE OF MAGNETOSPHERES

\begin{tabular}{lccc}
\hline \hline \multicolumn{1}{c}{ Planet } & $\begin{array}{c}B_{\text {Surface }} \\
(\mathrm{T})\end{array}$ & $\begin{array}{c}R_{\mathrm{PI}} \\
(\mathrm{km})\end{array}$ & $\begin{array}{c}R_{\mathrm{MP}} \\
\left(R_{\mathrm{Pl}}\right)\end{array}$ \\
\hline Earth $\ldots \ldots \ldots$ & $3.0 \times 10^{-5}$ & 6378 & 10 \\
Mercury $\ldots \ldots$ & $2.6 \times 10^{-7}$ & 2440 & 1.5 \\
Jupiter $\ldots \ldots \ldots$ & $9.6 \times 10^{-4}$ & 68700 & 55 \\
Saturn $\ldots \ldots \ldots$ & $4.9 \times 10^{-5}$ & 57550 & 25 \\
Uranus $\ldots \ldots \ldots$ & $9.1 \times 10^{-6}$ & 25050 & 18 \\
Neptune $\ldots \ldots$ & $1.8 \times 10^{-5}$ & 24700 & 26 \\
\hline
\end{tabular}




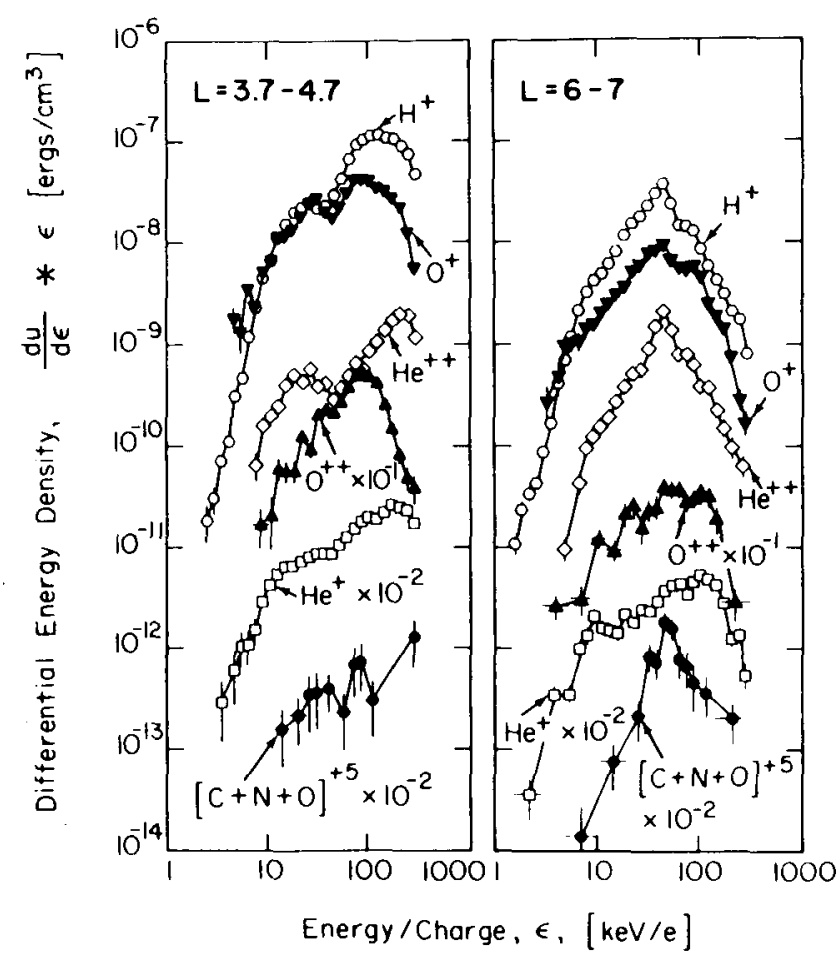

FIG. 2.-Differential flux of different ion species vs. energy/charge ( $E$ / $Q)$ as measured in the ring current during a magnetospheric storm by the AMPTE CHEM instrument (Gloeckler et al. 1985).

singly charged ions from multiply charged solar wind ions or vice versa. However, the observed charge state distribution cannot be explained with only one source present (Kremser et al. 1988). Both, the ionosphere and the solar wind, have to contribute simultaneously, although their contribution may vary significantly with solar and magnetospheric activity (Stokholm et al. 1989). It can be clearly seen that the spectra are similar in energy per charge, i.e., their peak is at the same energy per charge. At $L=6-7$, i.e., in the magnetic shell around the Earth whose distance from the center is 6-7 $R_{\mathrm{E}}$ at the equator, a peak in energy per charge at around $60 \mathrm{keV} / e$ is observed with the peak shifting to $\approx 100 \mathrm{keV} / e$ at $L=3.7-4.7$. The complement of sources and acceleration processes in the magnetosphere will have to satisfy these observations. We will present several key processes the presence of which has been established by observations and modeling.

\subsection{Extraction of Ionospheric Ions}

Ionospheric ions have to be lifted out of the upper layers of the Earth's atmosphere and accelerated from energies of less than $1 \mathrm{eV}$ in the beginning to several ten or even hundred $\mathrm{keV}$ in their final particle distribution. This requires several consecutive steps in which the ions first have to be extracted out of the ionosphere and then accelerated. A schematic view of the processes involved in the auroral zone and the typical particle distributions is presented in Figure 3.

The sources of these ions are found in the auroral zones and the cusp region over the polar ionosphere (e.g., Chappel 1988; Moore 1991). In the ionosphere the ions are generally gravitationally bound (especially $\mathrm{O}^{+}$), and therefore are unable to escape to higher altitudes. Because no significant electric fields can be maintained in the ionosphere alternate energization processes have to be employed here. Observations in the topside polar ionosphere have revealed many examples of socalled "conics." These ion velocity distributions feature a conical shape centered around the magnetic field direction, which indicates that the original thermal distribution of the ionosphere must have undergone a heating perpendicular to the magnetic field $B$ with part of the energy transferred into the upward direction parallel to $B$. The latter part of the process may be understood in terms of upwelling in the inhomogeneous polar magnetic field due to the grad $B$ force, in which the parallel energy of the ions will adiabatically increase at the expense of their perpendicular energy. As a result of these processes fairly flat "conics" emerge from the ionosphere at relatively low altitudes, the so called transversely accelerated ions (TAI), which were first observed by Sharp, Johnson, \& Shelley (1977). As the dominant free energy source for the production of these ion distributions lower hybrid waves driven by auroral energetic electrons have been proposed (e.g., Chang \& Coppi 1981; Retterer, Chang, \& Jasperse 1986). Recently the connection between TAI distributions and bursts of lower hybrid waves has been established observationally by Vago et al. (1992) at altitudes between 500 and $1000 \mathrm{~km}$ in the nightside auroral zone. The observations show wave activity in thin filamentary density cavities oriented along the magnetic field lines. This process may be seen as the ion feeder into the further acceleration in auroral arcs at higher altitudes which leads to more energetic ion "conics" with a much steeper cone angle than the TAIs.

\subsection{Acceleration Parallel to $B$ in the Auroral Zones}

From the observation of "Inverted $V$ " electron distributions below an auroral arc and more energetic ion conics as

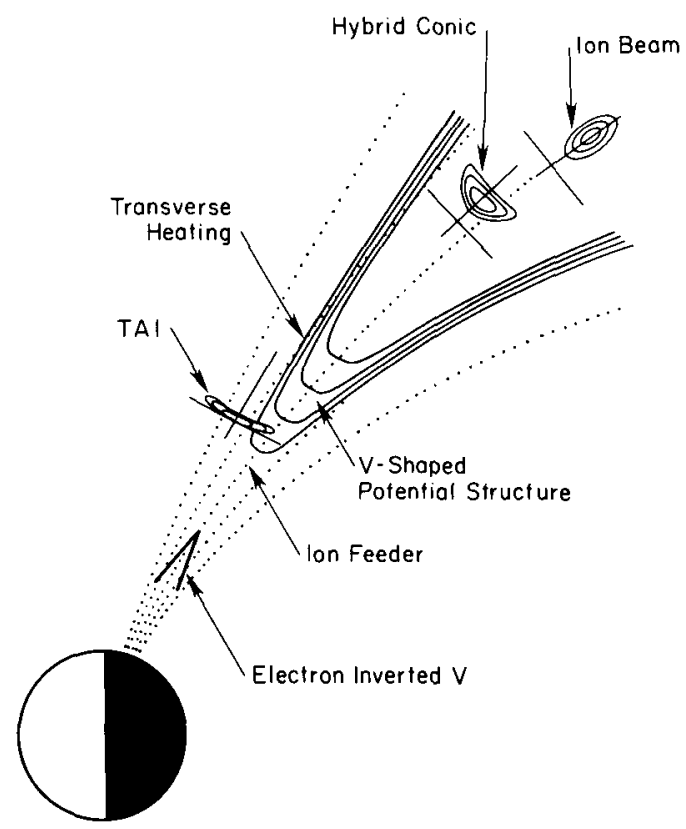

FIG. 3.-Schematic overview of the magnetic field configuration and the physical processes operative in the auroral zone (Shelley \& Collin 1991). 
well as ion beams above the auroral arc, the presence of a confined ( $V$-shaped) potential structure along the magnetic field lines has been suggested (e.g., Shelley \& Collin 1991, and references therein). Ions flowing out of the ionosphere in the way described above will enter the potential structure and then be accelerated by the electric field along the magnetic field lines. As a result ion beams with energies of a few $\mathrm{keV}$ are observed above auroral arcs.

That the energy is gained in a specific potential drop is confirmed by the observation of mixed $\mathrm{H}^{+}$and $\mathrm{O}^{+}$ion beams. During solar maximum, when the abundance of oxygen is high, both species reach the same total energy. During solar minimum when $\mathrm{O}^{+}$occasionally is only a minor species, plasma waves tend to accelerate $\mathrm{O}^{+}$towards the velocity of the $\mathrm{H}^{+}$ions, which still maintain the total energy provided by the potential drop underneath (Collin, Peterson, \& Shelley 1987; Reiff et al. 1988).

After this acceleration in the auroral zone ion beams are injected into the magnetotail, traveling along the magnetic field lines at the plasma sheet boundary where they merge with ions from the solar wind. Such ion beams have been reported as a quite frequent phenomenon. In particular, beams with ions of ionospheric and solar wind origin have been observed (Candidi, Orsini, \& Ghielmetti 1984) which flow in the same direction, but with slightly different velocities and cone angles.

\subsection{Acceleration in Magnetic Neutral Line Configurations}

The plasma sheet is one of the large storage regions of hot plasma and energetic particles in the magnetosphere. The energy spectra of energetic ions in the plasma sheet indeed reveal the presence of.both, ionospheric and solar wind ions, as can be seen in Figure 4 for quiet times (Gloeckler \& Hamilton 1987). The spectra of ion species within each group are identical; comparing the two groups, they are still very similar in energy per charge. Ionospheric ions show a somewhat harder slope and increased flux at low energies $(<5 \mathrm{keV}) . \mathrm{H}^{+}$is a mixture of both populations. Figure 5 shows a hardening of the energy spectra of both ionospheric and solar wind ion species in the plasma sheet in the course of a magnetospheric substorm (Möbius et al. 1987). The observed flux increases are best organized in energy per charge. These observations require an acceleration process which works in increments of energy per charge, before the ion population is injected into the plasma sheet. This again seems to suggest an acceleration through a finite electric potential.

As indicated above, a mixed population of ionospheric and solar wind ions flows along the magnetic field lines at the plasma sheet boundary in the tail. With the convection of these magnetic field lines toward the neutral sheet these ions enter also the magnetic neutral line region at the tailward edge of the plasma sheet. Here the ions become unmagnetized, and the cross-tail electric field, which is induced by the inward convection of the magnetic field lines, leads to a substantial energization of these ions (e.g., Speiser 1965, 1967; Büchner \& Zelenyi 1987). Finally, they are ejected again along the magnetic field lines into the earthward direction. A simple-minded description of this process seems to suggest an energization which adds increments of energy per mass due to the finite gyroradii effects involved. Based on an MHD simulation of magnetic
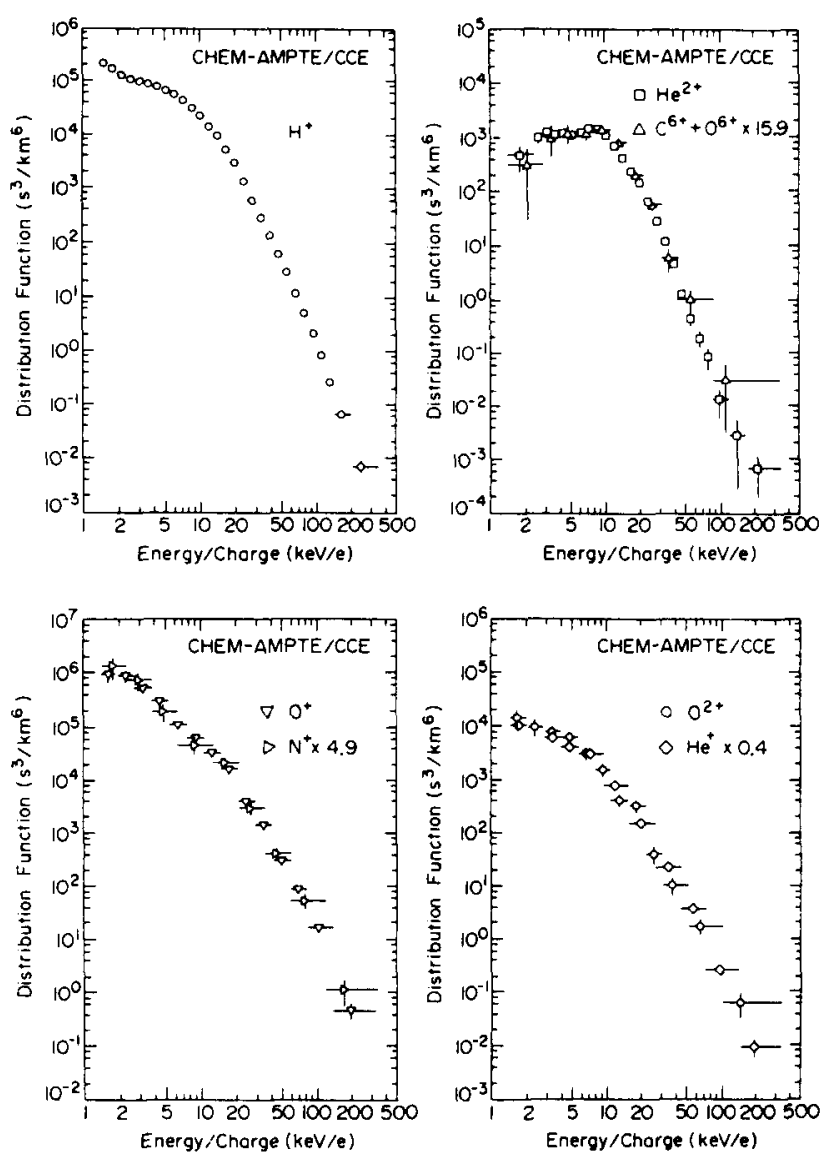

FIG. 4.-Differential flux of different ion species vs. energy/charge $(E /$ $Q$ ) as measured in the plasma sheet during quiet times by the AMPTE CHEM instrument (Gloeckler \& Hamilton 1987).

field reconnection in the tail (Scholer 1987) Sachsenweger et al. (1989) could reproduce energization which is organized in energy per charge. The temporal variation of the electric field during the reconnection process seemed to play an essential role in the acceleration to make it appear dependent on $E / Q$.

Various modeling approaches of the energization in the tail (see also Martin \& Speiser 1988) all seem to explain the energetic ion beams which are observed at the plasma sheet boundary (Möbius et al. 1980; Williams 1981). Scholer \& Jamitzky (1989) also simulated the energetic ion beams which are ejected from the neutral line into the tailward direction at the boundary of the plasmoid which leaves the tail after a substorm. However, the major shortcoming of these simulations still seems to be that none of them is able to reproduce the almost isotropic particle distributions observed in the central plasma sheet during both quiet and active times. It may be that additional scattering during the reconnection process, for example, in the possible case of turbulent reconnection (Ambrosiano et al. 1988) has not been included adequately.

The energetic particle population in the plasma sheet is the major source for the most energetic distributions in the inner magnetosphere. During magnetospheric substorms substantial numbers of energetic particles are injected onto dipolar field lines (McIlwain 1974). The ions then drift in the combined 


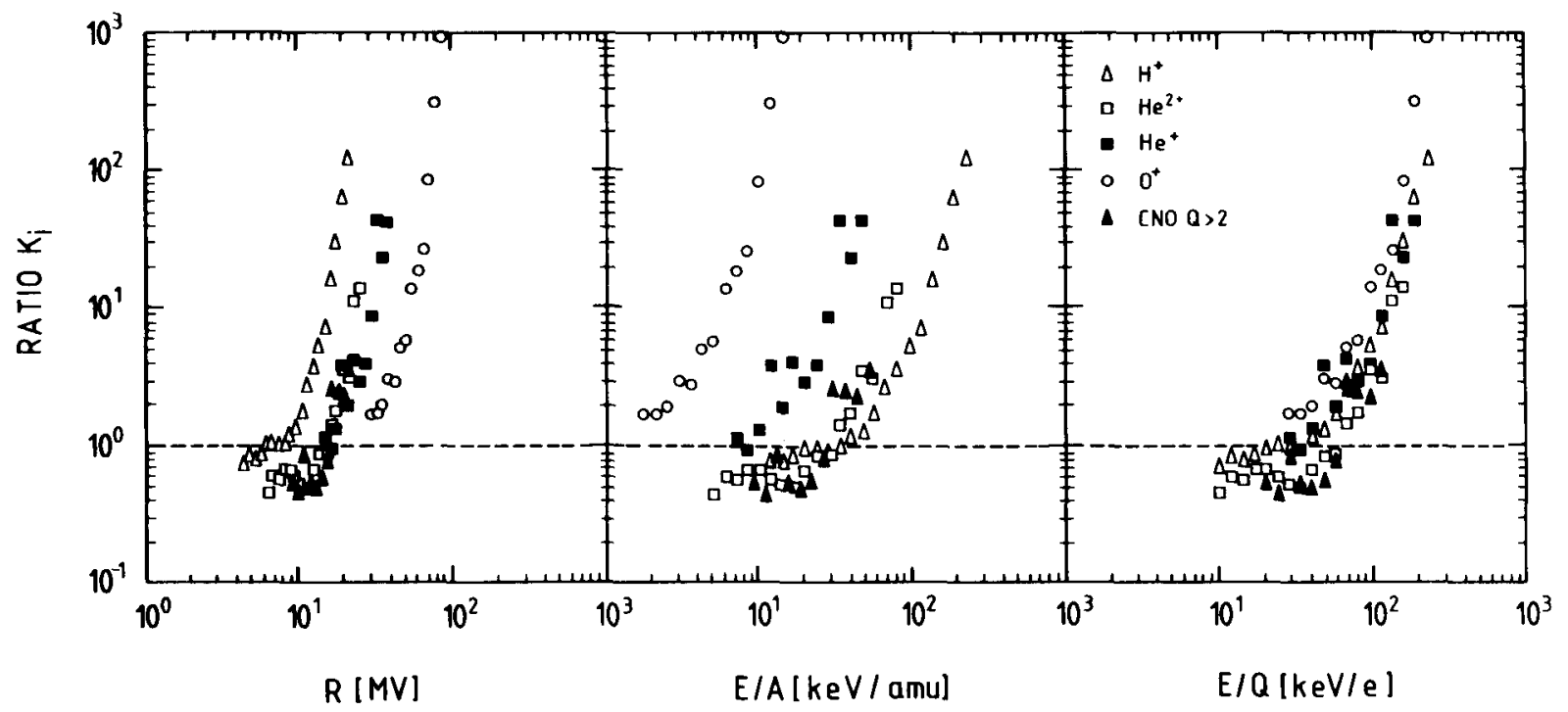

FIG. 5.-Ratios of the ion fluxes after and before substorm onset for $\mathrm{H}^{+}, \mathrm{He}^{2+}, \mathrm{He}^{+}, \mathrm{O}^{+}$, and $\mathrm{CNO}$ with $Q>2$ as a function of rigidity (left), energy/mass (center), energy/charge (right) (Möbius et al. 1987).

magnetic and electric fields to form the ring current. The interconnection between the tail and the ring current during substorms is discussed in more detail by Lopez (1994).

\subsection{Energization by Radial Diffusion in the Magnetosphere}

In the dipole field of the ring current regime the energetic particles are magnetically confined in a very efficient way. Therefore, this region of the magnetosphere is an excellent storage ring for energetic particle populations. Once on these field lines the particles tend to maintain their energy. Without any temporal disturbances the particles perform strictly adiabatic trajectories. For a detailed description of the particle behavior the reader is referred to the comprehensive presentation by Schulz \& Lanzerotti (1974), however, a brief overview is presented here.

The ions perform three basic motions which are connected to separate invariant quantities:

Gyration.-The ions gyrate about the magnetic field. For variations on time scales longer than the gyration time the first adiabatic invariant

$$
\mu=\frac{p_{\perp}^{2}}{2 m B}
$$

i.e., the magnetic moment of the particle, is conserved.

Bounce motion.-The ions are reflected back and forth in the increasing magnetic field at lower altitudes, the altitude depending on the pitch angle of the particle trajectory. For variations with timescales longer than the bounce time of a particle the second adiabatic invariant

$$
J=\int p_{\|} d s
$$

i.e., the average momentum parallel to the magnetic field, is conserved.

Drift motion.-Due to the curvature and the gradient of the magnetic field the energetic particles drift around the Earth in fixed drift shells in the dipole regime. The third adiabatic invariant associated with this motion is the total magnetic flux $\Phi$ enclosed by the drift orbit of the particle. For variations of the configuration on time scales longer than the drift time, the total magnetic flux and therefore the average total energy of the particle remains constant.

In response to variations of the kinetic pressure of the solar wind the magnetosphere reacts with quasi-static deformations of its magnetic field configuration. Because of asymmetries in these deformations and their short variation time in comparison with the drift time, particles will switch to a different drift shell. As a consequence the total magnetic flux seen by the particle will change. Since the magnetic moment of the particle remains conserved, its momentum $p_{\perp}$ perpendicular to the magnetic field will follow the field strength $B$ and thus the total energy of the particle will vary. The pressure pulses from the solar wind can be described as a randomized change of the third adiabatic invariant and thus lead to a random walk of the ring current particles. The major source of the particle distribution is the magnetotail, i.e., it is found at the outer boundary of the ring current. Therefore, a radial gradient of the particle density which points outward is maintained which causes an inward diffusion of the energetic ions in the presence of any magnetic irregularities. As a net result the particle population gains energy during the inward diffusion approximately proportional to the local magnetic field strength.

In logarithmic representation the shape of the energy spectra remains unchanged throughout this transport process, and the relative energization is the same, no matter whether described in $E / Q, E / M$, or $E$. Therefore, the ordering in $E / Q$, which is observed in the plasma sheet, is not altered after transport and further acceleration on the way into and within the ring current. In addition, the drift motion (gradient and curvature drift), which does alter the spectra, scales with $E / Q$ itself. This scenario also holds true in the most energetic regime, the radiation belts. 


\subsection{Additional Sources for Energetic Particles in the Inner Magnetosphere}

Despite the efficiency of the magnetospheric transport of ions the process described above is not the major source of the most energetic $(E>30 \mathrm{MeV})$ particle population in the inner radiation belt. The majority of the radiation belt protons in this energy regime are injected through a process known as Cosmic Ray Albedo Neutron Decay (CRAND) (e.g., Freden \& White 1960; Lavine \& Vette 1970). In collisions of cosmicray particles with atoms of the Earth's upper atmosphere neutrons are produced which then decay with a half-life of $\approx 10$ minutes into protons, electrons, and neutrinos. The protons are captured in the Earth's magnetic field and then undergo the adiabatic and nonadiabatic transport described above, thereby forming the most energetic population of the inner radiation belt.

Recently the contribution of yet another source to the ring current and radiation belt population, i.e., the anomalous cosmic rays (ACR), has been reported (Grigorov et al. 1991). These particles penetrate into the magnetosphere because of their high magnetic rigidity as singly ionized particles, undergo charge exchange to higher charge states, and then are stored for a long time.

\section{COMPARISON WITH OTHER MAGNETOSPHERES}

The basic characteristics of the ring current particle population are similar in all magnetospheres. It has been established that the energy spectra of all observed species can be best organized in terms of energy per charge in the Jovian (Hamilton et al. 1981) and the Saturnian magnetosphere (Hamilton et al. 1983 ). Furthermore, a similar positive radial gradient is observed in the phase space densities of energetic ions in the ring currents of Earth, Jupiter, Saturn, and Uranus. Figure 6 (from

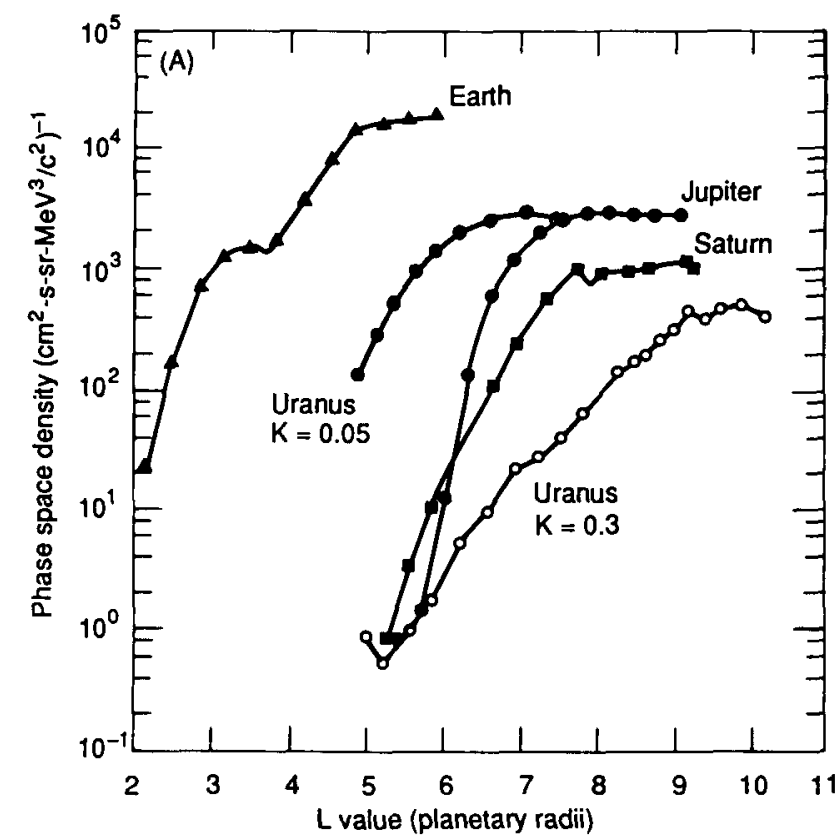

FIG. 6.-Phase space densities of ions with a rigidity of $100 \mathrm{MeV} \mathrm{G}^{-1}$ vs. distance from the planet ( $L$ in planetary radii) for Earth, Jupiter, Saturn, and Uranus (Cheng et al. 1987).
Cheng et al. 1987) shows the phase space densities of $\mu=100$ $\mathrm{MeV} \mathrm{G}^{-1}$ ions as a function of the distance $L$ in planetary radii from the planet. In all magnetospheres the density levels at a certain maximum beyond a distance of several planet radii, typically 5 for the Earth and about 8-9 for the other planets. Although the almost exponential decrease of the flux toward the planet seems to suggest a simple radial diffusion, it was shown (Haerendel 1968) that such profiles can only be reconciled by a combination of radial diffusion and a loss process which depends on the local phase space density of the particles.

The absolute values given in Figure 6 have been determined under the assumption that the vast majority of the ions are protons. This assumption is appropriate for the Earth and Uranus. However, Jupiter's magnetosphere contains a majority of heavy ions and Saturn's magnetosphere is thought to have a major contribution of heavy ions at high energies (Cheng et al. 1987). This leads to a significant upward correction of the phase space densities for Jupiter and Saturn which scale with $\mathrm{m}^{3 / 2}$. The density in the Jovian system turns out to be the highest followed by the Earth and Saturn, while the Uranian magnetosphere seems relatively depleted of energetic ions (Krimigis et al. 1986).

These observations support the idea that the basic scenario of the particle acceleration and transport is similar in all magnetospheres of the solar system. However, there are three aspects which modify this simple picture:

1. While the loss of energetic particles from the terrestrial ring current is dominated by charge exchange with exospheric atoms (Hamilton et al. 1988) with some contribution by wave particle interactions (e.g., Cornwall, Coroniti, \& Thorne 1970), the presence of moons in the inner magnetospheres of Jupiter, Saturn, Uranus, and Neptune add distinct sinks for the energetic particle distributions which lead to marked signatures in the radial profiles (Schardt \& Goertz 1983; Schardt \& McDonald 1983; Mauk et al. 1987; Mauk et al. 1991).

2 . The moons and rings within these magnetospheres constitute additional important sources for the particles in these magnetospheres.

3. The extension of particle populations corotating with the planetary magnetic field to larger distances from the planet alters the transport and acceleration patterns in some of the magnetospheres in comparison with the Earth's.

The latter two results will be discussed in more detail in the next section with reference to the Jovian magnetosphere.

\section{ENERGETIC PARTICLES IN THE JOVIAN MAGNETOSPHERE}

As already mentioned above, the energy spectra of all species in the inner Jovian magnetosphere are similar in energy per charge. Therefore, all spectra could be compiled into a single composite spectrum, assuming a reasonable charge state according to the origin of each ion species and after adjusting for the individual abundance ratios. This spectrum is seen in Figure 7 from Hamilton et al. (1981). Basically a similar sequence of acceleration and transport processes may be assumed for the Jovian magnetosphere as has been presented for the Earth. However, in addition to ions from the solar wind $\left(\mathrm{He}^{2+}, \mathrm{C}^{6+}\right)$ and the Jovian ionosphere $\left(\mathrm{H}^{+}, \mathrm{H}_{3}^{+}\right)$, ions such as $\mathrm{O}^{2+}$ and $\mathrm{S}^{+}$ indicate that Jupiter's moons, in particular Io, are a very strong ion source. These ions are accelerated to high energies within 


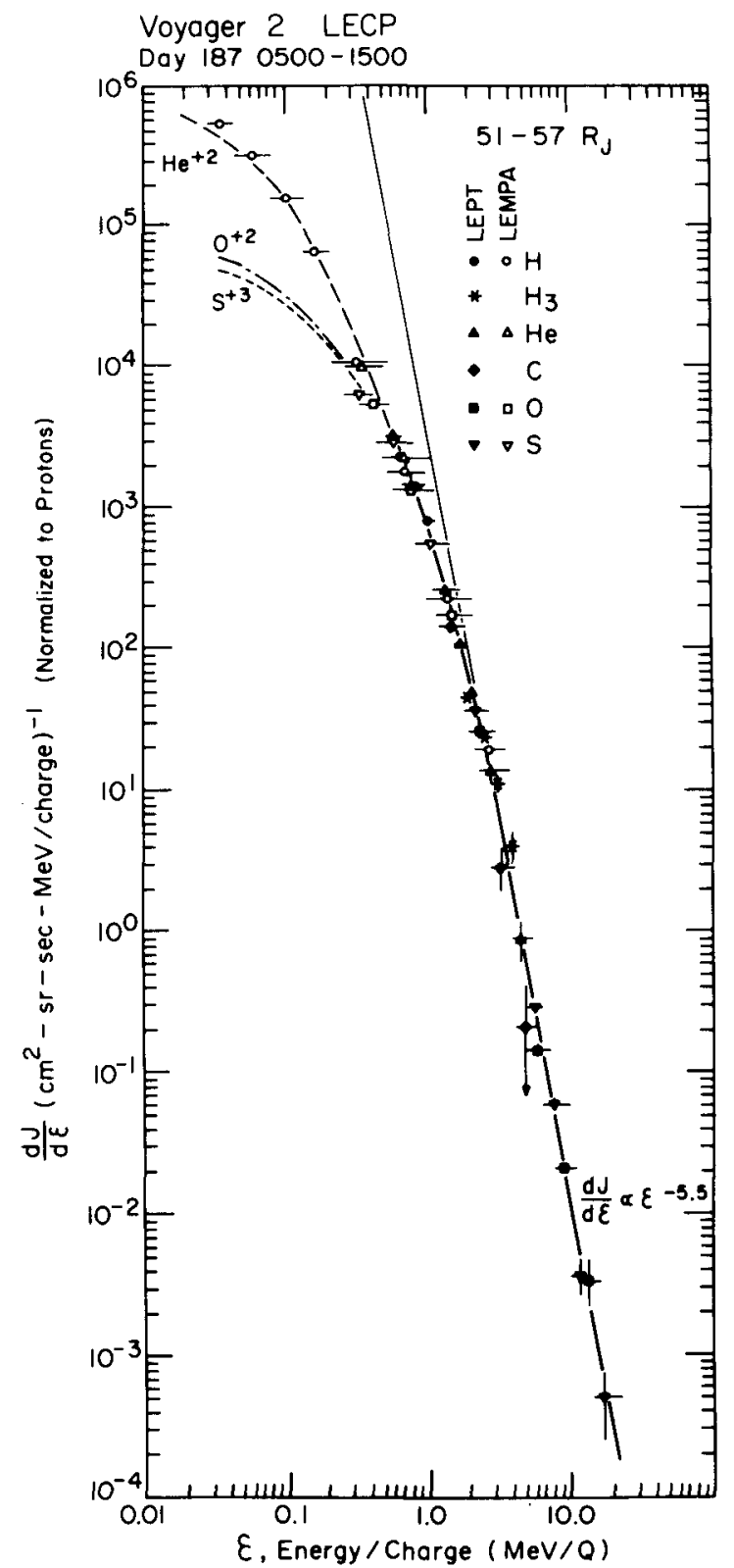

FIG. 7.-Energy spectra of various ion species in the inner magnetosphere of Jupiter as measured by the LEMPA and LEPT sensors on Voyager $I$ and 2 . The spectra have been displayed as a function of energy per charge assuming charge states of $\mathrm{H}^{+}, \mathrm{He}^{2+}, \mathrm{H}_{3}^{+}, \mathrm{C}^{6+}, \mathrm{O}^{2+}, \mathrm{Na}^{2+}, \mathrm{S}^{3+}$, and $\mathrm{Fe}^{12+}$ (Hamilton et al. 1981).

the magnetosphere, probably in the same way as those of solar wind and ionospheric origin.

It is important to note that no variation in the abundance ratios of the energetic particle population with distance from Jupiter has been observed throughout the inner magnetosphere. New time-of-flight instrumentation on the Ulysses spacecraft has now revealed that the composition of the source plasma on the contrary is highly variable throughout the magnetosphere. As can be seen in Figure 8, the $\mathrm{H}^{+} / \mathrm{He}^{2+}$ ratio has its maximum closest to the planet (Geiss et al. 1992), which can be interpreted as the variation of the relative importance of the solar wind and ionospheric source with distance from Jupiter.

The combination of the observations that (1) the abundance of the energetic particles is constant while the plasma sources vary with distance and (2) the phase space density of the ring current distribution shows a positive radial gradient leads to the conclusion that the ions of all sources must already be accelerated in the outer magnetosphere and then transported inward with further acceleration through radial diffusion. This can be readily understood in the same way as for the Earth's magnetosphere for the ionospheric and solar wind ion components, but it seems to pose a problem for ions from Jupiter's moons. The major sources of these ions, e.g., the moon Io, are located within the inner magnetosphere itself.

Although some of the sources are well inside the inner magnetosphere, a substantial flux of energetic ions from all potential sources is found at the outer boundary. This requires either a transport of a sufficient amount of plasma out into the outer magnetosphere and an acceleration of all ions in this regime, e.g., by reconnection electric fields (Carbary, Hill, \& Dessler 1976) as in the Earth's magnetotail, before they are transported inward again, or a recirculation of more energetic particles back into the outer magnetosphere (Nishida 1976). A way to preserve most of the energy they have gained on their inward diffusion would be to let these particles move to higher $L$-shells, e.g., close to the polar ionosphere under the influence of increased electric field fluctuations. An alternative way to bring material from the inner moons into the outer magnetosphere is charge exchange of corotating ions with stationary neutral gas in the vicinity of the satellites (Eviatar, Mekler, \& Coroniti 1976). The resulting fast neutrals will reach the outer magnetosphere basically unimpeded, where a large fraction will be reionized in the presence of energetic particles. Most of

$$
\mathrm{H}^{+} \text {and } \mathrm{He}^{2+}
$$

IN THE JOVIAN MAGNETOSPHERE

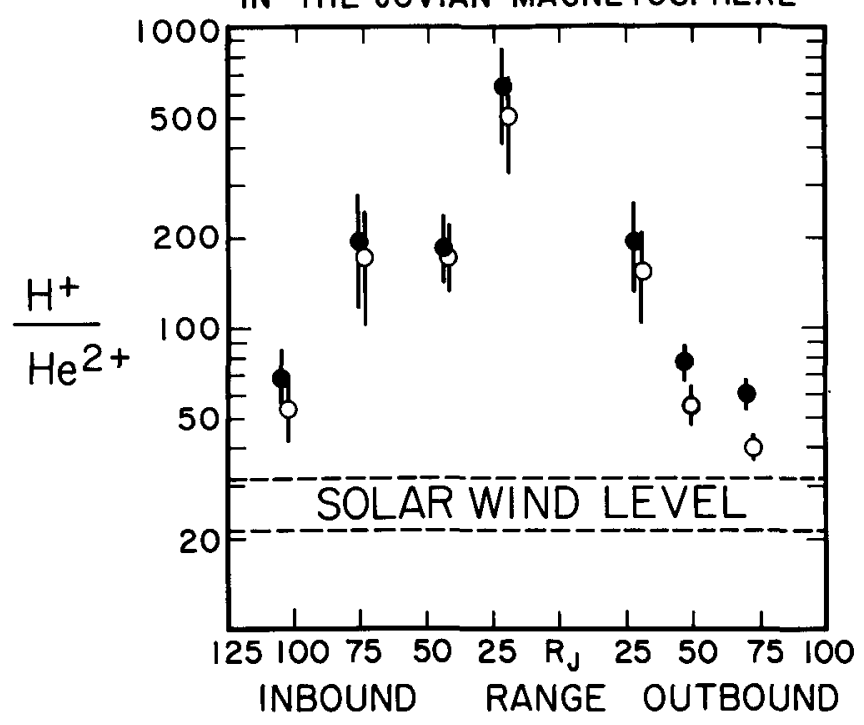

Fig. 8. $-\mathrm{H}^{+} / \mathrm{He}^{2+}$ ratios vs. distance from Jupiter during the Ulysses fly-by (Geiss et al. 1992). 
these suggestions to enhance the energization of the ion population in the Jovian magnetosphere in principle involve the same physical processes for acceleration as already discovered in the Earth's magnetosphere. No new process is added, except perhaps magnetic pumping (Goertz 1978), which may be seen as a more organized way to increase the energy of particles. While the particles drift around the planet they experience a sudden increase of the magnetic field once every rotation when entering the more compressed sector of the Jovian magnetosphere.

The strong contribution of corotation in Jupiter's magnetosphere is another significant difference when compared with the Earth. In the Earth's magnetosphere the plasma is corotating up to the plasmapause, beyond which the plasma motion is convection controlled driven by solar wind induced convection electric fields. However, in the Jovian magnetosphere the corotating regime reaches out to the magnetopause, because the electric field $E_{\text {cor }}$ induced by corotation is much stronger throughout the Jovian magnetosphere, than the solar wind induced convection field $E_{\mathrm{con}}$ at the distance $r$ of Jupiter from the Sun (Brice \& Ioannidis 1970). The corotation electric field:

$$
E_{\text {cor }}=B(R) \omega R
$$

depends on the planetary magnetic field $B$ at the distance $R$ from the planet and the planet's rotation frequency $\omega$. Therefore, the strong magnetic field and the fast rotation of Jupiter enhances the effects of corotation. The convection electric field:

$$
E_{\mathrm{con}}=\alpha B_{\mathrm{sw}}(r) v_{\mathrm{sw}}
$$

depends on the local interplanetary magnetic field $B_{\mathrm{sw}}$ and solar wind velocity $v_{\mathrm{sw}}$. The factor $\alpha$ denotes the efficiency with which the interplanetary magnetic field couples to the magnetosphere. Generally $\alpha=10 \%$ is taken as a good approximation. This significant modification of the magnetospheric transport pattern by corotation in the Jovian system makes possible more efficient combinations of the basic acceleration mechanisms, the quantitative effect of which still needs to be explored in future investigations.

This corotation presumably is also the background for the still unresolved clocklike modulation of the energetic electron population in the Jovian magnetosphere (McKibben \& Simpson 1974) and in interplanetary space. The modulation of the electron flux and of the slope of the electron spectrum is maintained precisely at the synodic rotation period of Jupiter, as has been reemphasized recently by the observations of the Ulysses spacecraft (Simpson et al. 1992). The phase of the variation matches precisely the extrapolation from earlier missions such as Pioneer 10, and 11 and the Voyagers. Dessler \& Hill (1975) proposed the so-called magnetic anomaly model as an explanation. In this model a magnetic anomaly leads to enhanced precipitation of energetic particles into a certain region of the ionosphere where the ionospheric conductivity is increased as a result. In turn this creates a more active sector of the magnetosphere with a profound influence on the magnetotail, where periodically-when the active sector points toward midnight -magnetospheric plasma and energetic electrons may be released.

\section{PARTICLE AND ENERGY SOURCES FOR ENERGETIC PARTICLE POPULATIONS IN MAGNETOSPHERES}

Let us now summarize the various aspects of particle acceleration and try to generalize them for all planetary magnetospheres. It was demonstrated that various plasma sources contribute to the energetic particle populations and that a sequence of acceleration processes is needed to produce the observed energy spectra. The observed and potential particle sources and acceleration processes are compiled in Table 2 for all planetary magnetospheres in our solar system. All particle populations finally undergo radial diffusion as a combined transport and acceleration process which ultimately determines the distributions in the ring current and radiation belts

TABLE 2

\begin{tabular}{|c|c|c|c|c|c|c|c|c|}
\hline \multirow[b]{2}{*}{ SOURCE } & \multirow[b]{2}{*}{ ACCELERATION } & \multirow[b]{2}{*}{ REGION } & \multicolumn{6}{|c|}{ Planet } \\
\hline & & & $\mathbf{M}$ & $\mathrm{E}$ & $\mathbf{J}$ & $\mathrm{S}$ & $\mathrm{U}$ & $\mathrm{N}$ \\
\hline \multirow[t]{3}{*}{ Ionosphere } & $E \| B$ & Aurorae & & $\mathrm{x}$ & I & I & I & I \\
\hline & Reconnection & Tail & & $\mathrm{x}$ & I & I & $\mathrm{x}$ & I \\
\hline & Radial diffusion & Inner magnetosphere & & $\mathrm{x}$ & $\mathrm{x}$ & $\mathrm{x}$ & $\mathrm{x}$ & $\mathrm{x}$ \\
\hline \multirow{2}{*}{ Solar wind } & Reconnection & Tail & $x$ & $\mathrm{x}$ & I & I & $\mathrm{x}$ & I \\
\hline & Radial diffusion & Inner magnetosphere & & $\mathrm{x}$ & $\mathrm{x}$ & $\mathrm{x}$ & $\mathrm{x}$ & $\mathrm{x}$ \\
\hline CRAND & Radial diffusion & Inner magnetosphere & & $\mathrm{x}$ & & $x$ & & \\
\hline $\mathrm{ACR}$ & Radial diffusion & Inner magnetosphere & & $\mathrm{x}$ & & & & \\
\hline \multirow[t]{2}{*}{ Moons .......... } & Reconnection & Tail & & & I & I & & 1 \\
\hline & Radial diffusion & Inner magnetosphere & & & $\mathrm{x}$ & $\mathrm{x}$ & & $\mathrm{x}$ \\
\hline \multirow[t]{2}{*}{ Rings $\ldots \ldots \ldots \ldots$} & Reconnection & Tail & & & & I & & \\
\hline & Radial diffusion & Inner magnetosphere & & & & $\mathrm{x}$ & & \\
\hline
\end{tabular}

ION SOURCES AND ACCEleration Mechanisms in PLANetary MagnetosPheres

NOTES._ " $x$ " indicates direct observational evidence of the particle source and/or acceleration process; "I" indicates inference. 
of the inner magnetospheres. Only the particles injected by CRAND or from the anomalous component of cosmic rays are energetic enough at the beginning that no additional acceleration process in the magnetosphere is required. The CRAND source seems to be consistent with the most energetic proton distribution in the Earth's magnetosphere (e.g., Hess 1968; Lavine \& Vette 1970), and Schardt \& McDonald (1983) have shown that a CRAND production at the rings is compatible with the observed proton distribution in the Saturnian magnetosphere. ACR ions have recently been reported in the Earth's magnetosphere (Grigorov et al. 1991). The remaining sources require initial acceleration in the outer magnetosphere which scales in $E / Q$ in order to explain the observed energy spectra. For most of the magnetospheres this step is indirectly inferred (marked with an "I" in Table 2) from the inability of pure radial diffusion to reproduce the observations. Only in the Earth's magnetosphere the individual steps have been tracked with measurements in all key regions.

As far as the energy sources for the acceleration processes are concerned, two different actors come in mind: (1) the convective electric field of the solar wind and (2) the corotation electric field due to the planetary rotation.

In Table 3 the effects of these two potential energy sources are expressed in terms of electric fields according to equations (5.1) and (5.2) for all magnetospheres. In addition, the total convective potential over the polar cap and the total energy achieved by corotation are given. While the magnetospheres of the Earth and Mercury are mostly convection dominated, Jupiter's and Saturn's are completely corotation dominated. In the Uranian and Neptunian magnetosphere both energy sources seem to contribute significantly. The relative importance of both depends strongly on the coupling coefficient $\alpha$ between solar wind and magnetosphere. Yet in terms of the total energy which may be achieved by applying the full potential, the solar wind is the dominating energy source. A pulsar magnetosphere has been added for comparison, in which case only rotation plays a role for particle transport and energization. There is no important external flow like the solar wind. In a pulsar magnetosphere the particles can in principle be accelerated up to the light cylinder. Therefore, the limitation of their energy gain is determined by relativistic effects, which are not part of this review. The entity closest to a pulsar magnetosphere in our solar system is the Jovian magnetosphere, where some of the features expected at pulsars may be studied in situ.

\section{FUTURE PERSPECTIVES IN MAGNETOSPHERIC PARTICLE ACCELERATION}

Much progress has been made in understanding magnetospheric acceleration and transport processes with satellites and spaceprobes. To date these studies have been limited even in the Earth's magnetosphere to single or two spacecraft measurements. The simultaneous determination of the large-scale transport of particle populations and the synoptic view of the important plasma processes with adequate spatial resolution have been hampered by the lack of coordinated satellite networks. The other magnetospheres in the solar system have been visited in short fly-bys which could only provide snapshots. Future studies of magnetospheric acceleration and transport will expand our understanding by removing these limitations.

The investigation of the Earth's magnetosphere will be set on a much broader base within the internationally coordinated approach of the ISTP (International Solar Terrestrial Program) which is being implemented over the next 4 years. Satellites will simultaneously monitor key regions of the magnetosphere: GEOTAIL (already in orbit) is devoted to the deep tail, the input into the magnetosphere from the solar wind is scanned by WIND and the polar magnetosphere is explored by POLAR (both to be launched in 1994). This program will be complemented in Summer of 1994 by the launch of the FAST (Fast Auroral Snapshot explorer) spacecraft, which will study the auroral zones with very high time and spatial resolution. In late 1995 or early 1996 the four CLUSTER spacecraft will start to focus on the three-dimensional structures of small-scale (several $100 \mathrm{~km}$ ) to medium-scale (several $1000 \mathrm{~km}$ ) structures in the magnetosphere and at its boundaries. Both FAST and CLUSTER, by emphasizing high time resolution (and thus high spatial resolution ) and simultaneous observations at four locations, respectively, will enhance our understanding of the detailed particle acceleration processes in the aurorae, in the magnetotail, and at the magnetospheric boundaries.

With the Galileo probe, which will reach Jupiter in 1994, the first systematic long time study of the Jovian magnetosphere becomes possible with a trajectory which includes multiple encounters with Jovian satellites. These repeated visits to the interaction regions with moons will also further our understanding of the injection of material from these satellites. A similar long time study of the Saturnian magnetosphere will

TABLE 3

ENERgy SOURCES For PARTICLE TRANSPORT AND ACCELERATION

\begin{tabular}{|c|c|c|c|c|c|c|c|}
\hline PlaneT & $\begin{array}{l}B_{\text {Surface }} \\
(\mathrm{T})\end{array}$ & $\begin{array}{l}\text { PERIOD } \\
(\mathrm{hr})\end{array}$ & \multicolumn{2}{|c|}{ SOLAR WIND } & \multicolumn{2}{|c|}{ Planet Rotation } & $\begin{array}{c}E_{\text {Cor }} / E_{\text {Conv }} \\
\text { MAGNETOPAUSE }\end{array}$ \\
\hline Mercury ...... & $2.6 \times 10^{-7}$ & 1406 & $1.3 \times 10^{-3}$ & 1.4 & $3.4 \times 10^{-7}$ & $4.1 \times 10^{-10}$ & 0.0 \\
\hline Jupiter ....... & $9.6 \times 10^{-4}$ & 9.5 & $9.6 \times 10^{-5}$ & 114.2 & $4.2 \times 10^{-3}$ & 11 & 43.7 \\
\hline Saturn ........ & $4.9 \times 10^{-5}$ & 10.1 & $5.3 \times 10^{-5}$ & 23.7 & $8.2 \times 10^{-4}$ & 1.4 & 15.6 \\
\hline Pulsar ........ & $1.0 \times 10^{+8}$ & $2.8 \times 10^{-5}$ & 0.0 & 0.0 & $2.8 \times 10^{+8}$ & $v \rightarrow c$ & (Only Rotation!) \\
\hline
\end{tabular}


follow with the arrival of CASSINI at Titan, probably in the year 2003.

By expanding the scope of investigation in Earth's magnetosphere with comprehensive and coordinated studies as well as the first long time investigation of the important Jovian and Saturnian magnetospheres, the door will be open to more detailed comparative magnetospheric studies. The knowledge acquired on acceleration processes over a wide parameter range will finally lead to a quantitative extrapolation of these results and to understanding particle acceleration in pulsar magneto- spheres and other astrophysical objects with strong intrinsic magnetic fields.

The work was supported under NASA grant NAG5-1548. The author is thankful to Johannes Geiss and George Gloeckler for providing presentation material for the review. Very helpful discussions with Gerhard Haerendel, Douglas C. Hamilton, Lynn M. Kistler, Dave Klumpar, Martin A. Lee, Kristina Lynch, and Donald G. Williams during the preparation of the review are gratefully acknowledged.

\section{REFERENCES}

Ambrosiano, J., Matthaeus, W. H., Goldstein, M. L. \& Plante, D. 1988, J. Geophys. Res., 93, 14383

Brice, N. M., \& Ioannidis, G. A. 1970, Icarus, 13, 173

Büchner, J., \& Zelenyi, L. M. 1987, Gerlands Beitr. Geophys., 96, 179

Candidi, M., Orsini, S., \& Ghielmetti, A. G. 1984, J. Geophys. Res., 89, 2180

Carbary, J. F., Hill, T. W., \& Dessler, A. J. 1976, J. Geophys. Res., 81,5189

Chang, T., \& Coppi, B. 1981, Geophys. Res. Lett., 8, 1253

Chappel, C. R. 1988, Rev. Geophys., 26, 229

Cheng, A. F., Krimigis, S. M., Mauk, B. H., Keath, E. P., MacLennan, C. G., Lanzerotti, L. J., Paonessa, M. T., \& Armstrong, T. P. 1987, J. Geophys. Res., 92, 15315

Collin, H. L., Peterson, W. K., \& Shelley, E. G. 1987, J. Geophys. Res., 92, 4757

Cornwall, J. M., Coroniti, F. B., \& Thorne, R. M. 1970, J. Geophys. Res., 75,4699

Dessler, A. J., \& Hill, T. W. 1975, Geophys. Res. Lett., 2, 567

Eviatar, A., Mekler, Y., \& Coroniti, F. V. 1976, ApJ, 205, 622

Freden, S. C., \& White, R. S. 1960, J. Geophys. Res., 65, 1377

Geiss, J., et al. 1992, Science, 257, 1535

Gloeckler, G., \& Hamilton, D. C. 1987, Phys. Scripta, 18, 73

Gloeckler, G., Wilken, B., Stüdemann, W., Ipavich, F. M., Hovestadt, D., Hamilton, D. C., \& Kremser, G. 1985, Geophys. Res. Lett., 12, 325

Goertz, C. K. 1978, J. Geophys. Res., 83, 3145

Grigorov, N. L., et al. 1991, Geophys. Res. Lett., 18, 1959

Haerendel, G. 1968, in Earth's Particles and Fields, ed. B. M. McCormac (New York: Reinhold), 171

Hamilton, D. C., Brown, D. C., Gloeckler, G., \& Axford, W. I. 1983, J. Geophys. Res., 88,8905

Hamilton, D. C., Gloeckler, G., Ipavich, F. M., Stüdemann, W., Wilken, B., \& Kremser, G. 1988, J. Geophys. Res., 93, 14343

Hamilton, D. C., Gloeckler, G., Krimigis, S. M., \& Lanzerotti, L. J. 1981 , J. Geophys. Res., 86, 8301

Hess, W. N. 1968, in Introduction to Space Science, ed. W. N. Hess \& G. D. Mead (New York: Gordon \& Breach), 179

Jones, F. 1994, ApJS, this issue

Kremser, G., Stüdemann, W., Wilken, B., Gloeckler, G., Hamilton, D. C. \& Ipavich, F. M. 1988, Ann. Geophys., 6, 325

Krimigis, S. M. 1994, in preparation

Krimigis, S. M., et al. 1986, Science, 233, 97

Lavine, J. P., \& Vette, J. I. 1970, Models of the Trapped Radiation Environment, Vol. 6: High Energy Protons (NASA SP-3024)

Lopez, R. 1994, ApJS, 90, 531

Martin, R. F., \& Speiser, T. W. 1988, J. Geophys. Res., 93, 11521

Mauk, B. H., Keath, E. P., Kane, M., Krimigis, S. M., Cheng, A. F., Acuna, M. H., Armstrong, T. P., \& Ness, N. F. 1991, J. Geophys. Res., 96, 19061

Mauk, B. H., et al. 1987, J. Geophys. Res., 92, 15283

Mcllwain, C. E. 1974, in Magnetospheric Physics, ed. B. McCormac (Dordrecht: Reidel ), 143

McKibben, R. B., \& Simpson, J. A. 1974, J. Geophys. Res., 79, 3545

Möbius, E., Ipavich, F. M., Scholer, M., Gloeckler, G., Hovestadt, D., \& Klecker, B. 1980, J. Geophys. Res., 85, 5143

Möbius, E., Scholer, M., Klecker, B., Hovestadt, D., Gloeckler, G., \& Ipavich, F. M. 1987, in Magnetotail Physics, ed. A. T. Y. Lui (Baltimore: Johns Hopkins Univ. Press), 231

Moore, T. E. 1991, Rev. Geophys., 29, 1039

Nishida, A. 1976, J. Geophys. Res., 81, 1771

Reiff, P. H., Collin, H. L., Craven, J. D., Burch, J. L., Winningham, J. D., Shelley, E. G., Frank, L. A., \& Friedman, M. A. 1988, J. Geophys. Res., 93, 7441

Retterer, J. M., Chang, T., \& Jasperse, J. R. 1986, J. Geophys. Res., 91 , 1609

Sachsenweger, D., Scholer, M., \& Möbius, E. 1989, Geophys. Res. Lett., 16,1027

Sagdeev, R. 1994, in preparation

Schardt, A. W., \& Goertz, C. K. 1983, in Physics of the Jovian Magnetosphere, ed. A. J. Dessler (New York: Cambridge Univ. Press), 157

Schardt, A. W., \& McDonald, F. B. 1983, J. Geophys. Res., 88, 8923

Scholer, M. 1987, J. Geophys. Res., 92, 12425

Scholer, M., \& Jamitzky, F. 1989, J. Geophys. Res., 94, 2459

Schulz, M., \& Lanzerotti, L. J. 1974, Particle Diffusion in the Radiation Belts, (New York: Springer)

Sharp, R. D., Johnson, R. G., \& Shelley, E. G. 1977, J. Geophys. Res., 82, 3324

Shelley, E. G., \& Collin, H. L. 1991, in Auroral Physics, ed. C. I. Meng, M. J. Rycroft, \& L. A. Frank (New York: Cambridge Univ. Press), 129 Simpson, J. A., et al. 1992, Science, 257, 1543

Smith, E. J., Wenzel, K.-P., \& Page, D. E. 1992, Science, 257, 1503

Speiser, T. W. 1965, J. Geophys. Res., 70, 4219

1967, J. Geophys. Res., 72, 3919

Spjeldvik, W. N., \& Fritz, T. A. 1978, J. Geophys. Res., 83, 1583

Stokholm, M., Balsiger, H., Geiss, J., Rosenbauer, H., \& Young, D. T. 1989, Ann. Geophys., 7, 69

Vago, J. L., Kintner, P. M., Chesney, S. W., Arnoldy, R. A., Lynch, K. A., Moore, T. E., Pollock, C. J. 1992, J. Geophys. Res., 97, 16935

Williams, D. J. 1981, J. Geophys. Res., 86, 5507 